SUBJECT AREAS:

ELECTRONIC PROPERTIES

AND DEVICES

CHEMICAL PHYSICS

Received

28 July 2014

Accepted

7 October 2014

Published

28 October 2014

Correspondence and requests for materials should be addressed to

H.W.Z.

(hongweizhu@ tsinghua.edu.cn)

\title{
Electro- and Magneto-Modulated lon Transport through Graphene Oxide Membranes
}

\author{
Pengzhan Sun' ${ }^{1}$ Feng Zheng' ${ }^{1}$ Kunlin Wang' ${ }^{1}$ Minlin Zhong' ${ }^{1}$ Dehai Wu² \& Hongwei Zhu ${ }^{1,3}$
}

\begin{abstract}
'School of Materials Science and Engineering, State Key Laboratory of New Ceramics and Fine Processing, Key Laboratory of Materials Processing Technology of MOE, Tsinghua University, Beijing 100084, China, ${ }^{2}$ Department of Mechanical Engineering, Tsinghua University, Beijing 100084, China, ${ }^{3}$ Center for Nano and Micro Mechanics, Tsinghua University, Beijing 100084, China.
\end{abstract}

The control of ion trans-membrane transport through graphene oxide (GO) membranes is achieved by electric and magnetic fields. Electric field can either increase or decrease the ion transport through GO membranes depending on its direction, and magnetic field can enhance the ion penetration monotonically. When electric field is applied across GO membrane, excellent control of ion fluidic flows can be done. With the magnetic field, the effective anchoring of ions is demonstrated but the modulation of the ion flowing directions does not occur. The mechanism of the electro- and magneto-modulated ion trans-membrane transport is investigated, indicating that the electric fields dominate the ion migration process while the magnetic fields tune the structure of nanocapillaries within GO membranes. Results also show that the ion selectivity of GO membranes can be tuned with the electric fields while the transport of ions can be enhanced synchronously with the magnetic fields. These excellent properties make $\mathrm{GO}$ membranes promising in areas such as field-induced mass transport control and membrane separation.

M aterials with nanopores and nanochannels have attracted significant research interest in recent years due to their potential applications in mass transport and nanofluidics ${ }^{1,2}$. Among them, carbon nanomaterials, e.g., carbon nanotubes, which can afford an almost frictionless transport of water and effective ion exclusion due to their hydrophobic inner graphitic walls and narrow diameters, are believed to have potential applications in areas such as drug delivery, biomimetic selective ion transportation, water desalination and energy harvesting ${ }^{3-10}$. Recently, a growing number of attentions have been paid on graphene ${ }^{11}$, which has also been demonstrated as an excellent barrier film ${ }^{12,13}$. Typically, graphene is impermeable to all the gases and liquids despite its only one-atom thickness ${ }^{14}$. However, after introducing nanopores with various shapes, sizes and functional groups into the matrix, the nanoporous graphene can act as excellent sieving for various gases and ions in solutions, showing promises in molecular sieving ${ }^{15,16}$ and water desalination ${ }^{17}$. Graphene oxide (GO), prepared by the oxidization and exfoliation of graphite, is another promising 2D nanomaterial with unique structures, and is easy to synthesize and scale up ${ }^{18,19}$. GO sheets can be viewed as graphene attached with oxygencontaining functional groups, resulting in a 2D network of $s p^{2}$ - and $s p^{3}$-bonded atoms $s^{20,21}$. After self-assembly, GO sheets are strongly held together through hydrogen bonds to form freestanding laminate with sufficient mechanical strength. The nanocapillaries, from the clustering of $s p^{2}$ regions within the C-O matrix across all stacking layers, are responsible for the transport of water and ions through GO membranes ${ }^{22-24}$. Recently, it has been demonstrated that the anomalous unimpeded permeation of water through GO membranes can be achieved because of the formation of ice bilayer within the interlayer spaces and its melting transition at the edges of the flakes ${ }^{22,25}$. It has also been revealed that the coordination of transition metal ions with the $s p^{3}$ matrix and the cation- $\pi$ interactions of main group cations with the $\pi$ clusters of GO sheets are responsible for the ion-selectivity of GO membranes ${ }^{24,26}$. All of these characteristics make GO membranes as promising candidates in many applications, such as water purification and ion separation.

In terms of ion trans-membrane transport through membranes with nanopores and nanochannels, the control of fluid flows is of crucial importance which can open new opportunities for water purification based on novel field-induced reverse osmosis ${ }^{27-34}$. Typically, there are two strategies to control the ion trans-membrane transportation: modulating the ion migration process in solutions and altering the structure of nanochannels. In order to modulate the ion migration process, an external electric field can be applied across the membrane. Previous experimental and theoretical results have also demonstrated that unusual magnetic properties of room-temper- 
ature ferromagnetism along with weak antiferromagnetism ${ }^{35-43}$ are present in defective graphene films, based on which the alteration of the structure of nanocapillaries may occur with a magnetic field across GO membranes during ion transport processes, just like the magnetic field-induced realignment of carbon nanotubes and graphene $^{44,45}$.

In this work, the electro- and magneto-controlled ion trans-membrane transport properties of GO membranes are investigated based on the penetration of $\mathrm{KCl}, \mathrm{MgCl}_{2}, \mathrm{CaCl}_{2}$ and $\mathrm{FeCl}_{3}$. After the ion transport processes, Auger electron spectroscopy (AES) is used to determine the concentration of residual ions within GO membranes. Additionally, the mechanism on the electro- and magneto-induced ion transport through GO membranes is discussed.

\section{Results}

Characterizations of GO membranes. GO flakes were synthesized with the modified Hummers' method from potassium permanganate, sodium nitrite and concentrated sulfuric acid ${ }^{18,19}$. Figure la shows an AFM image of the as-synthesized GO sheets, which reveals that GO flakes have a lateral size of $\sim 1 \mu \mathrm{m}$. The inset in Figure 1a demonstrates the monolayer nature of the asprepared GO sheets. Freestanding GO membranes were prepared from the drop-casting of $\mathrm{GO}$ colloidal suspension $(1.5 \mathrm{mg} / \mathrm{mL}$, $\sim 1 \mathrm{~mL}$ ) onto a piece of smooth paper. After dried thoroughly, they were peeled off as shown in Figure $1 \mathrm{~b}$, which reveals the sufficient mechanical strength for freestanding manipulation (see Experimental Section and Figure S1). Figure S2 illustrates the cross-section of the as-prepared GO membrane, from which it can be evaluated that the as-prepared GO membranes possess a thickness of $1 \sim 2 \mu \mathrm{m}$. Figures $1 \mathrm{c}-\mathrm{d}$ are SEM images of the surface and crosssection of GO membranes, showing that the as-prepared GO membranes have a wrinkled surface and a lamellar cross-section structure.
Ion permeation experiments in the presence of both concentration gradients and external fields. The ion trans-membrane transport experiments were conducted on a self-made penetration apparatus (see Experimental Section, Figures 1e-f). Freestanding GO membranes were sealed with two-sided copper tape onto a plate with an aperture ( $\sim 5 \mathrm{~mm}$ in diameter), which separated the source vessel from the drain vessel. $80 \mathrm{~mL}$ of certain salt solutions $(0.1 \mathrm{~mol} / \mathrm{L})$ and deionized water were injected into the source and drain vessels, respectively. The conductivity variations of the drains were measured on a conductivity meter to reflect the penetrations of certain sources based on the fact that the conductivity is proportional to the concentration in diluted solutions. During the ion penetrations through GO membranes, electric and magnetic fields were applied as shown in Figures le and $f$, which enables us to investigate the electro- and magneto-induced ion trans-membrane transport properties of GO membranes.

The electro-induced ion transport through GO membrane is illustrated in Figure 2a and the conductivity variations of the drains for $\mathrm{KCl}$ sources are plotted in Figure 2b. It is found that a forward electric field (source to drain) leads to the increment of penetration while the backward electric field (drain to source) decreases the penetration. In contrast, with a magnetic field $(50 \mathrm{mT})$ on both directions across $\mathrm{GO}$ membranes as illustrated in Figure $2 \mathrm{c}$, the penetrations of $\mathrm{KCl}$ are increased significantly as shown in Figure 2d. The same experiments have been done based on the penetrations of $\mathrm{MgCl}_{2}$ and $\mathrm{CaCl}_{2}$ sources, and similar results are obtained as shown in Figure S3. Additionally, the applied magnetic fields are further varied on the direction from drain to source. Conductivity variations of the drains for $\mathrm{KCl}, \mathrm{MgCl}_{2}$ and $\mathrm{CaCl}_{2}$ are plotted in Figures $\mathrm{S} 4 \mathrm{a}-\mathrm{c}$, which reveal that ion penetrations are increased gradually with the enhancement of magnetic field. These results demonstrate that the ion transport through GO membranes can be either increased or decreased with
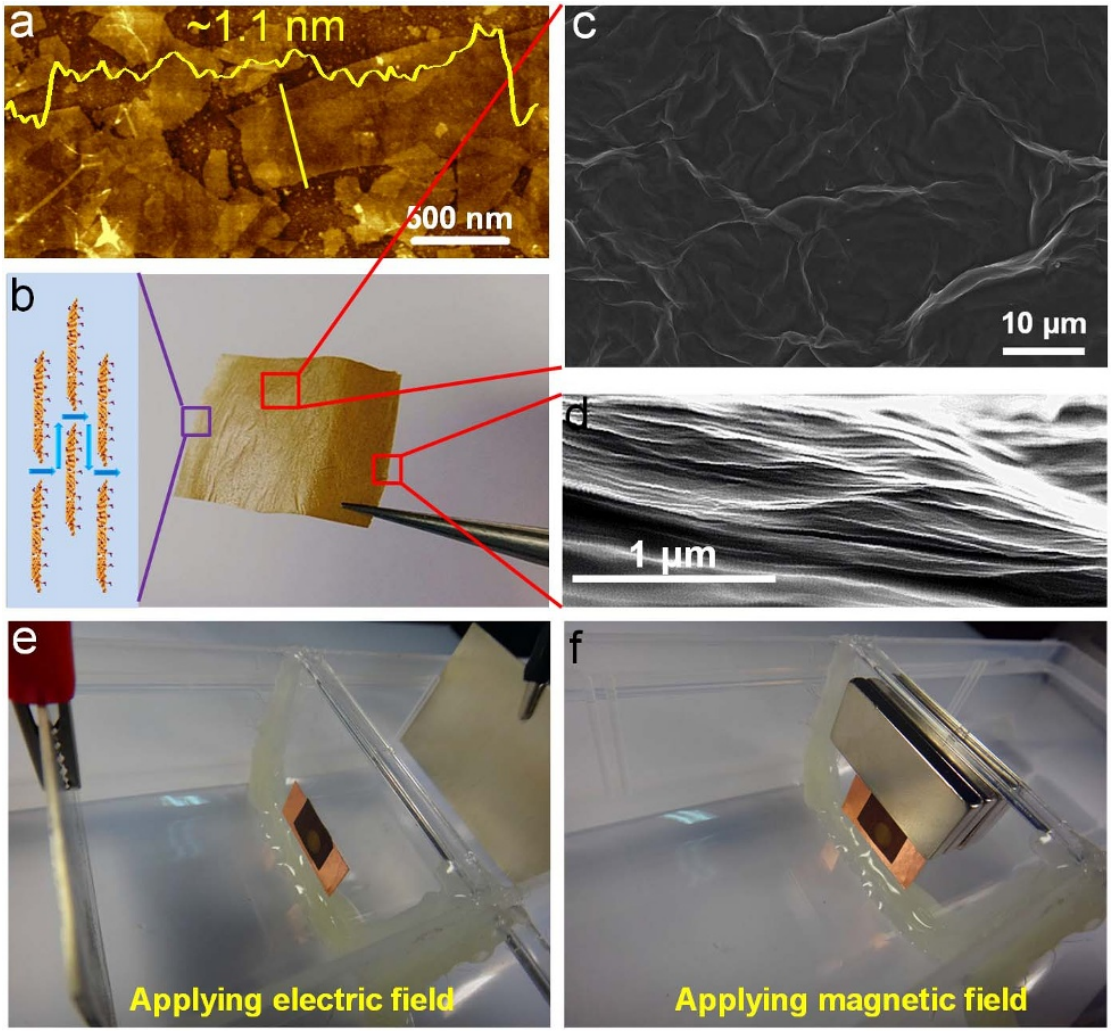

Figure 1 | (a) AFM characterization of as-prepared GO sheets. (b) Photograph of the as-prepared free-standing GO membrane. (c,d) SEM characterizations of the GO membrane. (e,f) Experimental apparatus for ion transport through GO membranes when applying the electric and magnetic fields. 

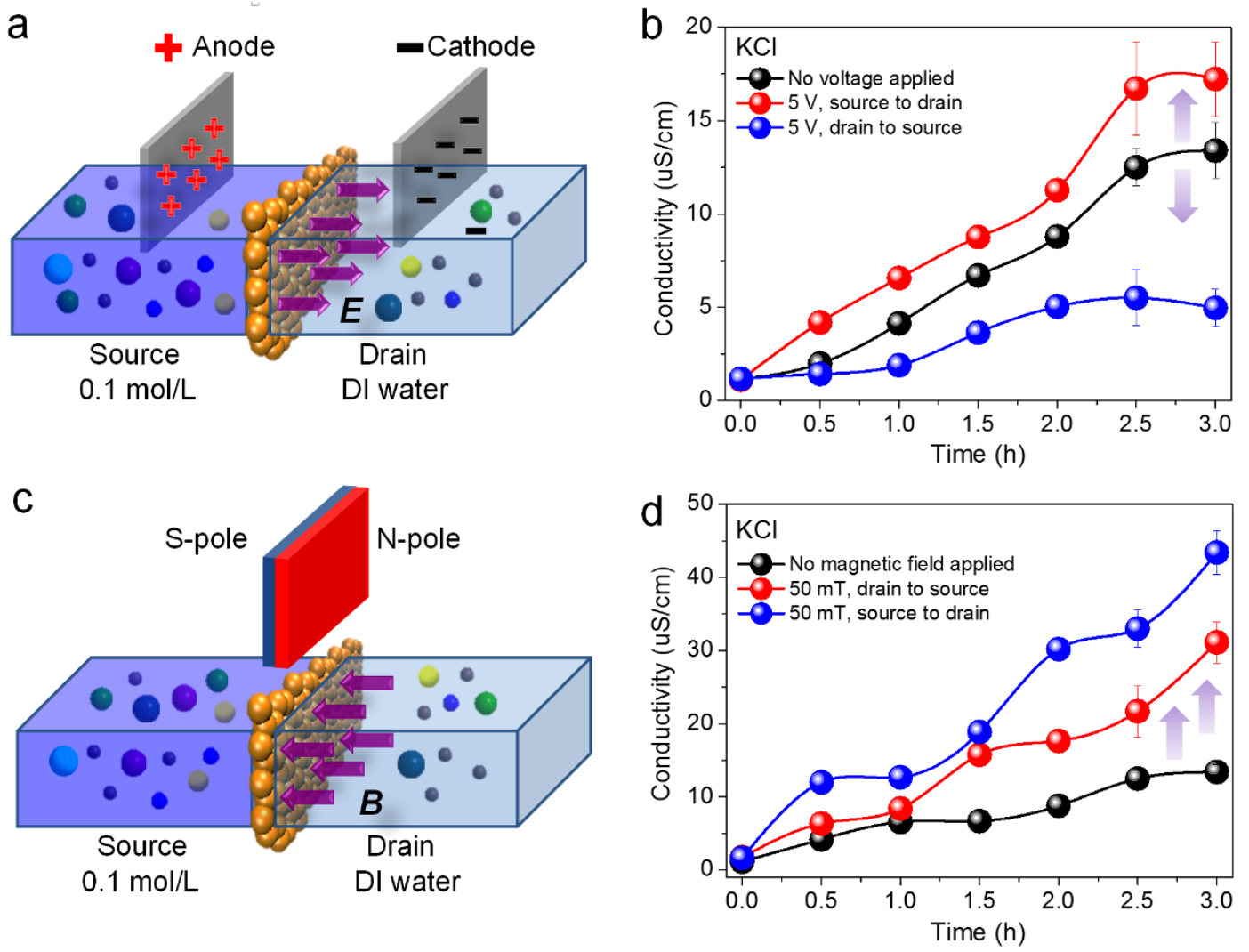

Figure $2 \mid$ (a) Schematic diagram of ion transport through GO membranes when applying electric field. (b) The conductivity variations of the drains for $\mathrm{KCl}$ sources when applying forward and backward voltages. (c) Schematic diagram of ion transport through GO membranes when applying magnetic field. (d) The conductivity variations of the drains for $\mathrm{KCl}$ sources when applying magnetic fields with opposite directions. All of the experiments were repeated for 3 times and the data points were calculated by averaging all of the corresponding data from the same series of experiments. The error bars show the largest fluctuating ranges for the corresponding data points.

electric field, which is depended on its direction. However, the magnetic field can only enhance the ion penetrations through GO membranes.

Ion permeation experiments in the presence of only external fields. The control of ion fluidic flows through GO membranes within electric and magnetic fields is further investigated, as shown in Figure 3. $80 \mathrm{~mL} 0.01 \mathrm{~mol} / \mathrm{L} \mathrm{KCl}$ solutions were injected into the source and drain vessels at the same velocity to ensure that no concentration gradients were present between source and drain solutions. During the penetration, an electric field or a magnetic field was applied across the GO membrane (Figures $3 a$ and c) and the conductivity variations of the sources and drains were measured. With an electric field across GO membrane (from source to drain) as illustrated in Figure 3a, obvious control of the ion flowing direction (from source to drain) occurs, which results in the increase of drain conductivity and the decrease of source conductivity (Figure $3 \mathrm{~b}$ ). If the voltage across GO membrane is reduced from 5 to $3 \mathrm{~V}$ (Figure S5), the modulation of the ion flowing direction is weakened gradually, indicating that the increase of electric field results in the enhancement of ion fluidic control. Interestingly, according to the conductivity variations in Figure $3 \mathrm{~b}$, it can be concluded that the decrease in source conductivity is larger than the increase of the drain when an electric field across GO membrane is applied. This asymmetrical change in conductivities might be attributed to the anchoring of ions around oppositely charged electrodes as well as around GO membranes. In contrast, with magnetic field across GO membrane (Figure 3d), conductivities of the source and drain are decreased significantly during the initial $1 \mathrm{~h}$ of penetration while maintain nearly unchanged afterwards. Curves of the conductivity variations in source and drain are nearly coincided together, indicating that no control of the ion fluidic directions occurs with the applied magnetic fields. Thus, it can be concluded that significant changes in ion transport through GO membranes occur with electric and magnetic fields. However, the electric fields cause effective modulation of ion flowing directions, while the magnetic fields cannot.

Ion permeation experiments based on $\mathrm{FeCl}_{3}$ solutions and external magnetic fields. Specially, the magneto-modulated transportation of $\mathrm{FeCl}_{3}$ through $\mathrm{GO}$ membranes is investigated and the results are shown in Figures S6,7. The reason why we chose to study the magneto-modulated permeance of $\mathrm{FeCl}_{3}$ is that the intense hydrolysis of $\mathrm{Fe}^{3+}$ would lead to the coexistence of abundant $\mathrm{Fe}^{3+}$ and $\mathrm{H}^{+}$ions in solutions, which facilitates the investigation of trans-membrane permeation of transition metallic $\mathrm{Fe}^{3+}$ and $\mathrm{H}^{+}$ions synchronously.

As shown in Figure S6, it reveals that when applying a magnetic field across the GO membrane with both directions, the penetrations of $\mathrm{FeCl}_{3}$ are enhanced (Figure S6a). Furthermore, increasing the strength of the magnetic field applied (from 25 to $50 \mathrm{mT}$ with the direction of drain to source) leads to the gradual increase of penetration through GO membranes (Figure S6b). The insets in each figure show the accurate concentrations of $\mathrm{Fe}^{3+}$ in drains after penetration for $3 \mathrm{~h}$ carried out by atomic emission spectroscopy, revealing that the trans-membrane transport of $\mathrm{Fe}^{3+}$ can be enhanced significantly by the magnetic fields applied. These results are similar to the cases of $\mathrm{KCl}, \mathrm{MgCl}_{2}$ and $\mathrm{CaCl}_{2}$, as shown in Figures 2, S3 and S4.

In addition to conductivity measurements, the $\mathrm{pH}$ values of the drains were also recorded during the application of magnetic fields, 

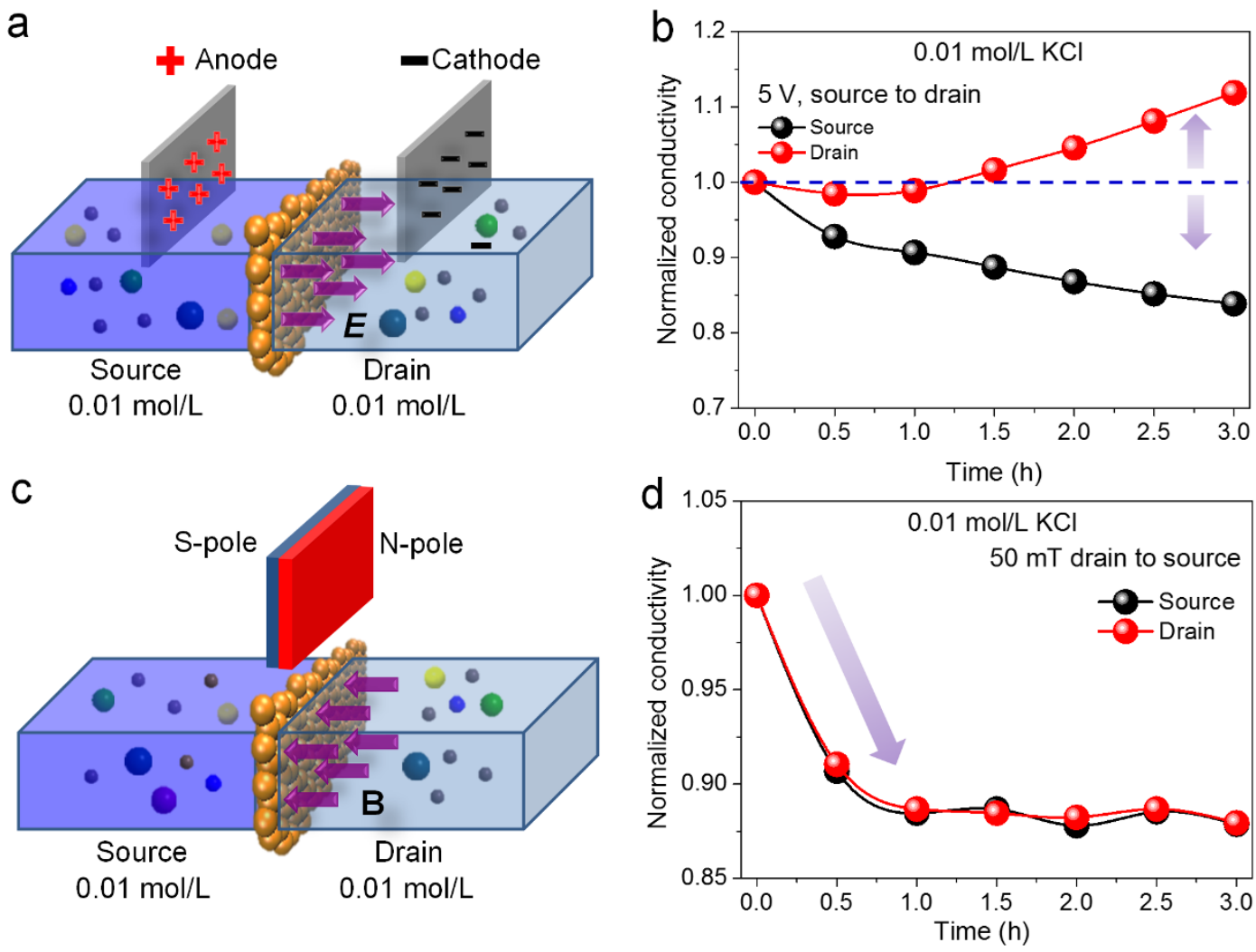

Figure $3 \mid$ (a) Schematic drawing of the control of ion fluidic flows through GO membranes in the presence of electric fields. (b) The conductivity variations of the source and drain under the condition of applying $5 \mathrm{~V}$ voltage (source to drain) when the source and drain are both injected with $0.01 \mathrm{~mol} / \mathrm{L} \mathrm{KCl}$. (c) Schematic drawing of the apparatus used to investigate the control of ion fluidic flows through GO membranes when applying magnetic fields. (d) The conductivity variations of the source and drain under the condition of applying $50 \mathrm{mT}$ magnetic field (drain to source) when the source and drain are injected with $0.01 \mathrm{~mol} / \mathrm{L} \mathrm{KCl}$ solutions.

as shown in Figures S6c-d. Surprisingly, it reveals that the transmembrane transport of protons is not affected by the magnetic field applied. The results in Figure S6 further imply that the transportations of metallic cations and $\mathrm{H}^{+}$ions through $\mathrm{GO}$ membranes should follow rather different mechanisms, which enables the effective separation of protons from $\mathrm{Fe}^{3+}$ cations during the trans-membrane permeation of iron-based electrolytes.

In order to investigate the different magnetic effects on the transport of $\mathrm{Fe}^{3+}$ and $\mathrm{H}^{+}$ions through GO membranes, the experiments of modulating the ion fluidic flows were done based on $\mathrm{FeCl}_{3}$ solutions and a $50 \mathrm{mT}$ magnetic field, as illustrated in Figure 3c. During the penetration, the conductivity and $\mathrm{pH}$ variations of the source and drain were both measured with time, as shown in Figure S7. It reveals that the conductivities of the source and drain are both decreased with penetration while the $\mathrm{pH}$ values are not changed under the condition of magnetic field applied. As the penetration of protons is not affected when applying a magnetic field across the GO membrane (Figure S7b), it can be concluded that the decrease of conductivities in source and drain (Figure S7a) is attributed to the magnetic-modulated trans-membrane transport of $\mathrm{Fe}^{3+}$.

Control experiments with cellulose microfilters and AES characterizations on GO membranes. To further reveal the tuning mechanism of electric and magnetic fields on the ion transmembrane transport through GO membranes, control experiments were conducted with commercial cellulose microfilters $(\sim 200 \mathrm{~nm}$ in aperture, $\sim 80 \%$ in porosity, as shown in Figure S8). With $5 \mathrm{~V}$ voltage along the direction from source to drain across the microfilter (Figure S8a), the conductivity variations of source and drain show similar change tendencies as in the cases of GO membranes, which indicates that electric fields play a major role in tuning the ion migration. However, the effect of modulating the ion flowing directions through GO membrane is greater than that through microfilter, which is presumably attributed to the diverse interactions of cations and anions within GO membranes. In detail, in aqueous environment, the ionization of oxygen functionalities decorated on GO should charge the GO membranes negatively. Besides the external electric fields, the electrostatic attractions and the cation- $\pi$ interaction $s^{26}$ between the cations and GO membranes can act as extra driving forces for the modulation of ion fluidic flows through GO membranes, which are absent in the typical polymer-based microfilters, further leading to the greater effect of modulating ion flowing directions in the case of GO membranes than microfilters. In contrast, when a $50 \mathrm{mT}$ magnetic field is applied along the direction from drain to source across the microfilter (Figure S8c), the conductivities of the source and drain are not changed markedly compared to that from GO membrane (Figure S8d), demonstrating that the magnetic field is ineffective to dominate the ion migration behavior in solutions but it might play an important role in altering the internal structure of GO membranes. The differing effects of electric and magnetic fields on the transmembrane transport of ions through GO membranes can also be found from the AES characterizations of GO membranes after ion penetrations, as shown in Figure S9. When an electric field is applied across the GO membrane, the concentration of residual cations within GO membranes is increased significantly (Figure S9b), indicating that the forward electric field enhances the ion flow through GO membrane to force more ions to interact with the GO nanocapillaries, which results in a larger amount of cations remained within GO membranes. However, in the case of magnetic field, the concentration of cations remained within GO membrane is reduced slightly (Figure S9c). Because the magnetic field across the GO 
membrane causes significant changes in ion penetration (Figures 2 and 3) while it has negligible effects on ion migration in solutions (Figure S8), it can be deduced that magnetic fields can alter the internal stacking structure of GO membranes to result in an enhanced transport of ions, while the electric fields only dominate the ion migration process in solutions and have negligible effects on GO membrane.

\section{Discussion}

Mechanism. The mechanism on the ion trans-membrane transport through GO membranes with external electric and magnetic fields is discussed in the following. In a typical aqueous solution, the hydrated ions are rearranged to reach an equilibrium state with two opposite forces; the electrostatic interaction leads to the formation of a partially ordered ionic cloud in which each ion (central ion) is surrounded by several oppositely charged ions; on the other hand, the random thermal jigging of ions disturbs the partially ordered structure of ionic cloud (Figure 4a). When an external electric field is applied, cations and anions will be accelerated in opposite directions and the symmetry of ionic cloud will be damaged (Figure 4a). Due to the opposite migrations of ionic clouds and central ions, the asymmetrical ionic clouds will hinder the transport of central ions and the central ions tend to attract the ionic clouds in the contrary direction. The electric force $F$ can be calculated as the following:

$$
F=E q=U q / d
$$

where $U$ is the voltage applied across GO membranes, $d$ is the distance between two planar electrodes and $q$ is the charge of ion.

In this study, $U$ is $5 \mathrm{~V}, d$ is $\sim 10 \mathrm{~cm}$ and the electric force $F$ of monovalent ions can be calculated as $8.0 \times 10^{-18} \mathrm{~N}$. On the other hand, the radius of ionic cloud can be expressed as the Debye screening length $\kappa^{-1}$ and the electrostatic interaction $F$ ' between monovalent cations and anions can be calculated according to the following equation,

$$
F^{\prime}=e_{0}{ }^{2} \kappa^{2} / 4 \pi \varepsilon_{r} \varepsilon_{0}
$$

where $e_{0}$ is the unit charge; $\kappa^{2}=2 e_{0}^{2} N_{\mathrm{A}} \rho_{\mathrm{s}} m^{0} I / \varepsilon_{r} \varepsilon_{0} k_{\mathrm{B}} T$, where $N_{\mathrm{A}}$ is the Avogadro's number, $\rho_{\mathrm{s}}$ is the density of solvent, $m_{0}=1 \mathrm{~mol} / \mathrm{kg}, I$ is the ionic strength, $\varepsilon_{r}$ is the relative permittivity, $\varepsilon_{0}$ is the vacuum dielectric constant, $k_{\mathrm{B}}$ is the Boltzmann constant and $T$ is the temperature.

At $25^{\circ} \mathrm{C}$, the electrostatic interaction $F^{\prime}$ between monovalent cations and anions in aqueous solutions $\left(\varepsilon_{r}=78.54, \rho_{\mathrm{s}}=997 \mathrm{~kg} / \mathrm{m}^{3}\right)$ can be evaluated as $\sim 3.18 \times 10^{-12} \mathrm{~N}$, which is six orders of magnitude greater than the electric force $F$ with external electric fields. Therefore, the central ions cannot be separated from their ionic clouds and only the relative ion transport rates and directions can be adjusted with the external electric fields. In terms of ion trans-membrane transport, due to the ionization of oxygen-containing functional groups in aqueous environment, the GO membranes would be negatively charged, resulting in the attraction of cations and repulsion of anions. The cations would firstly migrate into and through GO membranes and interact with the negatively charged GO nanocapillaries, while the anions would be attracted electrostatically by the cations into the drains as shown in Figure $4 \mathrm{~b}$. Therefore, the forward electric fields can accelerate the migration of cations and the backward electric fields hinder the transport of cations, while the anions would always be dragged passively by the electrostatic attractions from the cations to penetrate through GO membranes. Typically, the ion transport in electrolyte solutions and across GO membranes can be described by the extended Nernst-Planck equations:

$$
J_{i}=-D_{i} \frac{d C_{i}}{d x}-D_{i} \frac{F z_{i} C_{i}}{R T} \frac{d \varphi}{d x}+v C_{i}
$$

In this equation, $D_{i}$ is the diffusion coefficient, $F$ is the Faraday constant, $\varphi$ is the electric potential and $v$ is the linear convective velocity along the direction $x$. The three terms in Eq. 3 represent three mass transportation modes across GO membranes: (1) diffusion driven by concentration gradients; (2) diffusion by electric potential gradients; (3) convection by pressure gradients. This equation cannot be solved directly, but it can be used for explaining the modulation of ion transport through GO membranes in the presence of both concentration gradients and external electric fields qualitatively. In detail, in the presence of both a forward concentration gradient and a forward electric field across the GO membrane (the terms 1 and 2 in Eq. 3 describing the diffusions driven by concentration gradient and electric potential gradient possess the same sign), the diffusion driving force and the electric force should reinforce each other to result in an enhanced ion transport through GO membranes. On the other hand, in the presence of a forward concentration gradient and a backward electric field across the GO membrane (the terms 1 and 2 in Eq. 3 describing the diffusions driven by concentration gradient and electric potential gradient possess opposite signs), the diffusion driving force and the electric force should work against each other to result in a weakened ion transport through GO membranes, as demonstrated in Figure $2 \mathrm{~b}$. When the source and drain are both filled with $0.01 \mathrm{~mol} / \mathrm{L}$ electrolyte solutions and an electric field is applied across the GO membranes, only the electric driving force is present across the GO membrane and the electro-induced ion transport would be controlled by the inherent properties of ions (e.g. mobilities $u_{\mathrm{i}}$, charges, sizes and masses). With the radius of ions in Table $\mathrm{S} 1$, the ion mobility $u_{\mathrm{i}}$ can be calculated as the following:

$$
u_{\mathrm{i}}=z_{\mathrm{i}} e_{0} / 6 \pi \eta r_{\mathrm{i}}
$$

where $z_{\mathrm{i}} e_{0}$ is the charge of ion, $\eta$ is the viscosity of solvent and $r_{\mathrm{i}}$ is radius of the hydrated ion.

The external electric forces and the electrostatic attractions from GO membranes would drive the transport of cations from source to drain against the electrostatic hindrance from the anions, while the anions would be dragged by the cations to penetrate through GO membranes passively against the opposite external forces. After the cations and anions penetrate through the GO membranes and reach the drains, the migration of ions would be influenced by their inherent characters. In $\mathrm{KCl}$ solutions, due to the same monovalent charges and similar sizes of the hydrated $\mathrm{K}^{+}$and $\mathrm{Cl}^{-}$(Table S1), the mobilities of hydrated $\mathrm{K}^{+}$and $\mathrm{Cl}^{-}$are comparable (Eq. 4) and the external electric forces (Eq. 1) are equal in opposite directions, which result in the slow diffusion of $\mathrm{K}^{+}$and $\mathrm{Cl}^{-}$in drains and the delayed aggregation of ions around the cathode, and further the obvious control of ion fluidic flows for $\mathrm{KCl}$ solutions (Figure $3 \mathrm{~b}$ ). When GO membranes are changed to microfilters (Figures S8a and b), the relative changes of ion concentrations in the source and drain are much smaller, which can be attributed to the lack of electrostatic interaction between the ions and microfilters.

Previous experimental and theoretical studies have demonstrated the existence of prominent ferromagnetic along with antiferromagnetic features in most of graphene samples, presumably due to the presence of sheet defects and edge states ${ }^{3-42}$. The magnetic properties of GO membranes, which are composed of stacked and overlapped monolayer GO sheets, are also investigated in this study as shown in Figure 4c. Obviously, a dominant diamagnetism is evident in our GO samples at $300 \mathrm{~K}$ (demonstrated by the linear relation with inverse proportion between mass magnetization $M$ and $H$ ). After subtracting the diamagnetic background, as shown in the inset of Figure $4 \mathrm{c}$, it reveals that a weak room-temperature ferromagnetism is coupled in the GO membranes (demonstrated by the hysteresis characteristic $^{46}$ between excess moment $\Delta M$ and $H$ shown in the inset of Figure 4c). Therefore, based on the magnetic properties of GO membranes shown in Figure 4c, we conclude that the dominant diamagnetic accompanied with weak ferromagnetic behavior is pre- 
a
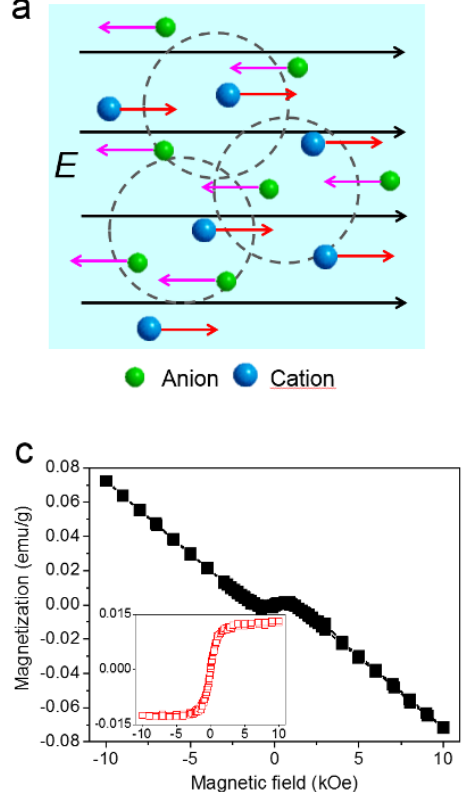

b

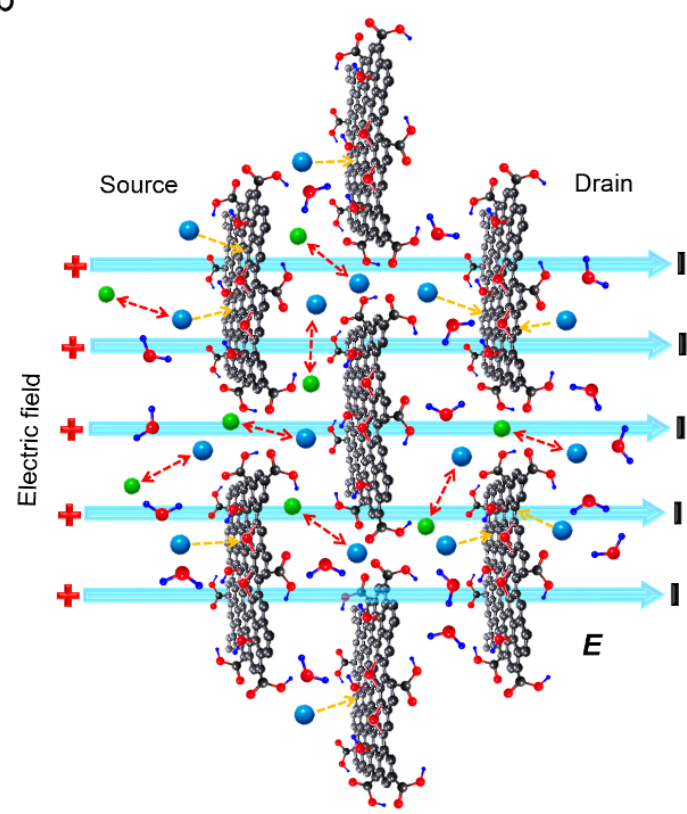

d

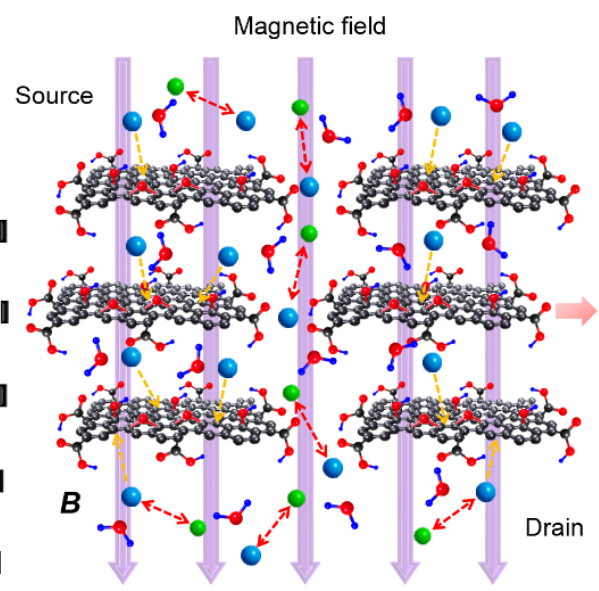

Electrostatic interaction

Cation-m interaction

Figure 4| (a) Diagrammatic sketch for the formation and motions of the ionic clouds under electric fields. (b) Schematic diagram of the mechanism proposed for the electro-induced ion trans-membrane transport through GO membrane. (c) $M-H$ curves of the GO membrane used in the experiments. The inset shows the excess moment $\Delta M$ as a function of $H$ of GO membrane after subtracting the diamagnetic background, showing the ferromagnetic component. (d) Schematic diagram of the mechanism proposed for the magneto-induced ion trans-membrane transport through GO membrane.

sent in GO membranes, which is in contrary to most of previous studies ${ }^{36,37,40-42}$. Presumably, the emergence of dominant diamagnetism is due to the inherent structural defects in graphene crystals ${ }^{46}$, while the room-temperature ferromagnetism is attributed to the external introduction of various defects such as adatoms, point defects, functionalities and magnetic edge states ${ }^{35-43,47}$. When a magnetic field across GO membrane is applied, due to the presence of room-temperature ferromagnetism in our GO sample, as shown in the inset of Figure $4 c$, the GO sheets within the membrane would be stacked more orderly ${ }^{44,45}$, as illustrated in Figure $4 \mathrm{~d}$. The more ordered stacking of GO sheets can shorten the length of GO nanocapillaries, which should facilitate the smooth trans-membrane transport of ions within the GO membrane (Figure $4 \mathrm{~d}$ ). Therefore, the magnetic field would lead to the enhancement of ion penetration through GO membranes (Figures 2, S3 and S4). During the magnetoresponse process, the GO flakes can be considered as numerous weak ferromagnetic bodies and the flake realignment process is independent of the direction of the magnetic field applied, further leading to the phenomenon that the magneto-modulated ion trans-membrane permeation is independent of the field direction (Figures 2, S3 and S6). Additionally, the shortening of GO nanocapillaries would also reduce the concentrations of cations remained within the membranes, which is in agreement with the AES results in Figure S9c. When $0.01 \mathrm{~mol} / \mathrm{L}$ certain electrolytes are injected into both the source and drain (Figure 3c), due to the strong attractions of metallic cations and the electrostatic repulsions of anions with the negatively charged GO membranes ${ }^{24,26}$, ordered double electric layers would be formed on the GO interfaces and the ions would be anchored tightly around the GO membranes, which might result in the decrease of ion concentrations in the main source and drain (Figure $3 \mathrm{~d}$ ). In contrast, for the transport of protons through GO membranes, the rapid migration of protons relies on the fast propagation through the hydrogen-bonding networks along the water layers formed in between the GO flakes ${ }^{48}$, which shouldn't be affected seriously by the more ordered stacking of GO sheets. Therefore, the permeation of protons is not changed by the magnetic field applied, as shown in
Figures S6c and d. In the case of ion trans-membrane transport through microfilters, the changes of ion concentrations in the source and drain are not obvious, compared to GO membranes, presumably due to the absence of strong interactions between ions and microfilters (Figures S8d).

Electro- and magneto-modulated trans-membrane transport of hybrid sources. Furthermore, the transport properties of hybrid sources through GO membranes under electric and magnetic fields are studied (illustrated in Figures $2 a$ and $c$ ). The concentrations of ions in drains after $3 \mathrm{~h}$ of penetration were quantified with atomic emission spectroscopy and liquid chromatography as shown in Figures 5 and 6. With electric fields across GO membranes, it is found in Figure 5 that, for most hybrid sources except $\mathrm{KCl}-\mathrm{K}_{2} \mathrm{SO}_{4}$, the forward electric field increases the ion penetration while the backward electric field decreases the penetration. Exceptionally for $\mathrm{KCl}-\mathrm{K}_{2} \mathrm{SO}_{4}$, the forward electric field weakens the ion transport through GO membranes (Figure $5 \mathrm{c}$ ), presumably due to the fact that the higher negatively charged $\mathrm{SO}_{4}{ }^{2-}$ would experience greater drags in the presence of forward electric fields and the penetrations of $\mathrm{SO}_{4}{ }^{2-}$ would be hindered markedly. On the other hand, the strong attraction between $\mathrm{SO}_{4}{ }^{2-}$ and $\mathrm{K}^{+}$ions would also slow the transmembrane transport of $\mathrm{K}^{+}$significantly, as demonstrated in Figure 5c. Notably, the selectivity of GO membranes towards different ions can be tuned with the electric fields on different directions. For example, in the case of $\mathrm{KCl}-\mathrm{NaCl}$ (Figure 5a), the penetration of $\mathrm{K}^{+}$is preferred with forward electric fields while $\mathrm{Na}^{+}$ is more favored with backward electric fields. For $\mathrm{KCl}-\mathrm{CaCl}_{2}$ (Figure 5b), the penetration of $\mathrm{K}^{+}$can be tuned significantly while the penetration of $\mathrm{Ca}^{2+}$ is not affected by the electric fields applied. In the cases of $\mathrm{KCl}-\mathrm{K}_{2} \mathrm{SO}_{4}$ and $\mathrm{KCl}-\mathrm{Na}_{2} \mathrm{SO}_{4}$ hybrid sources (Figures $5 \mathrm{c}$ and $\mathrm{d}$ ), the penetrations of anions through GO membranes are strongly influenced by the electric fields. The forward electric field favors the transport of $\mathrm{Cl}^{-}$while the ratio of $\mathrm{SO}_{4}{ }^{2-}$ ions is enhanced in the presence of backward electric field. In contrast, when magnetic fields are applied across GO membranes, the penetrations of cations and 

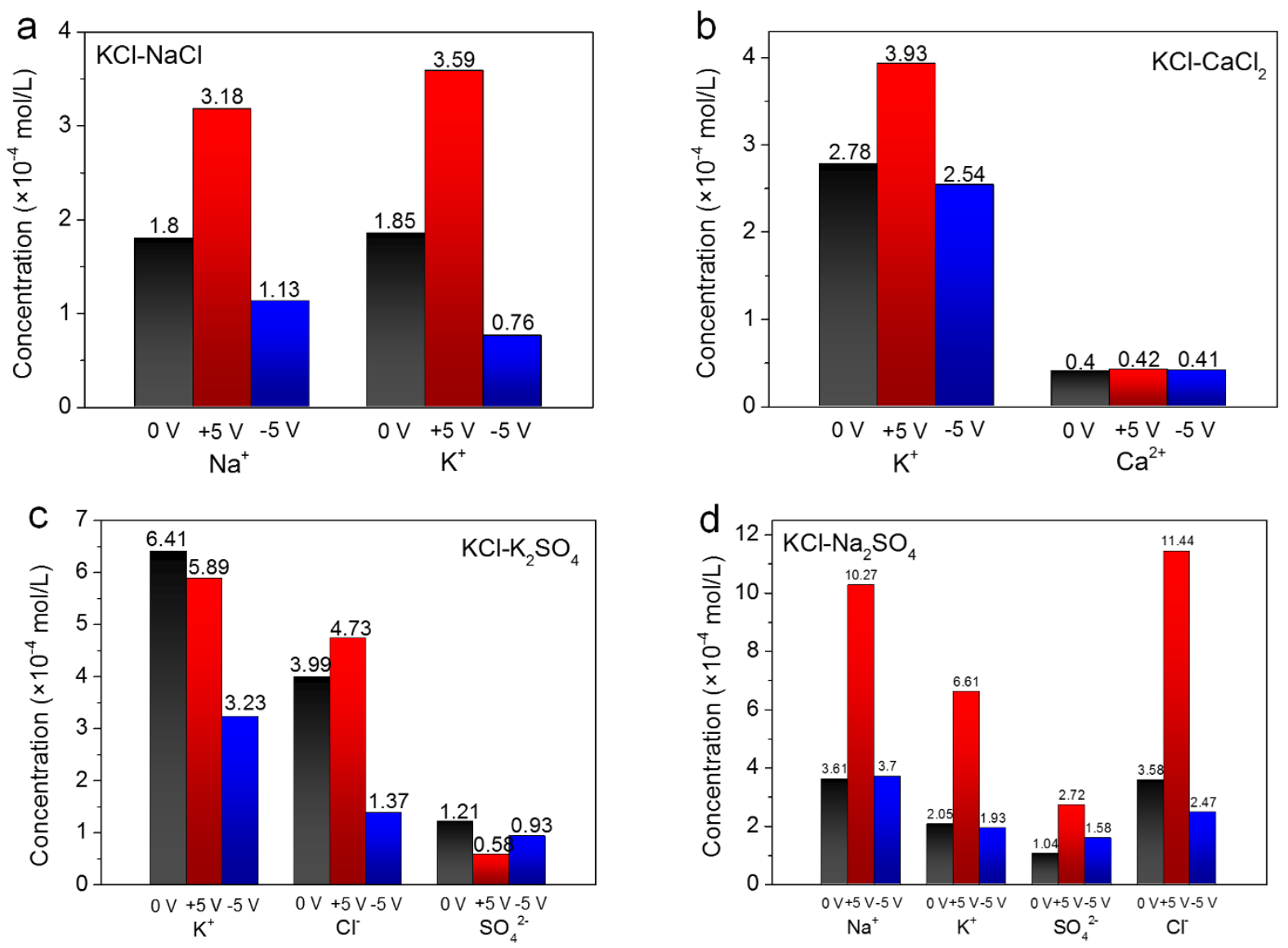

Figure 5 Ion concentrations of the drains during the penetrations of (a) $\mathrm{KCl}-\mathrm{NaCl}$, (b) $\mathrm{KCl}-\mathrm{CaCl}_{2},(\mathrm{c}) \mathrm{KCl}-\mathrm{K}_{2} \mathrm{SO}_{4}$ and (d) $\mathrm{KCl}_{-} \mathrm{Na}_{2} \mathrm{SO}_{4}$ hybrid sources when applying electric fields with opposite directions.

anions are enhanced synchronously and the selectivity towards different ions is not changed significantly as shown in Figure 6.

\section{Conclusion}

In summary, the control of ion trans-membrane transport through GO membranes has been achieved by electric and magnetic fields. It is found that the electric field can either increase or decrease the transport of ions depending on different directions, while the magnetic field can enhance the ion penetration through GO membranes only. Significant control of ion fluidic flows occurs when electric fields are applied across GO membranes while that does not occur with magnetic fields. Furthermore, mechanism of the electro- and magneto-induced ion trans-membrane transport is investigated, demonstrating that the electric fields dominate the ion migration in solutions while the magnetic fields alter the structure of nanocapillaries in GO membranes. Finally, the transport properties of hybrid sources through GO membranes with electric and magnetic fields are studied, revealing that the electric fields can adjust the selectivity of GO membrane towards different ions while the magnetic fields can enhance the ion transport synchronously. These excellent properties of GO membranes make them promising in areas such as field-induced mass transport control and membrane separation.

\section{Experimental Section}

GO membrane preparation. The monolayer GO flakes were prepared by the modified Hummers' method from potassium permanganate, sodium nitrite and concentrated sulfuric acid according to the previous methods ${ }^{18,19}$. After that, the asprepared GO flakes were dispersed in deionized water by sonication to form the $1.5 \mathrm{mg} / \mathrm{mL}$ GO colloidal solutions. Then we took a piece of label paper (shown in Figure S1a) and peeled off all the labels. The remaining smooth paper underneath was finally utilized for the subsequent GO membrane formation, as shown in Figure S1b.
Several droplets of GO solutions $(\sim 1 \mathrm{~mL})$ were drop-casted onto this smooth paper, as illustrated in Figure S1c. After that, the as-drop-casted GO droplets were left to dry spontaneously in air atmosphere, followed by detaching off from the smooth paper to form the free-standing GO membranes, as shown in Figure S1d.

Penetration experiments. The ion trans-membrane transport experiments were conducted with a home-made penetration apparatus (Figures le and f). The penetration apparatus was composed of two vessels (source and drain) separated by a plastic plate. An aperture with a diameter of $5 \mathrm{~mm}$ was located in the center of the plastic plate. A piece of free-standing GO membrane was sealed with double-sided copper tape (introducing a hole with the same diameter of $5 \mathrm{~mm}$ in the center) onto the aperture on the plastic plate so that the solutions in sources and drains could be directly connected by GO membranes to facilitate ion trans-membrane transport without passing through any bulky supports. The electric and magnetic fields were applied as illustrated in Figures 1e and f. For the application of electric fields, a pair of silver electrodes ( $99.99 \%$ in purity) was fixed across the GO membrane with a distance of $\sim 10 \mathrm{~cm}$ and voltages were applied between the silver electrodes (Figure 1e). For the application of magnetic fields, several pairs of magnets (Nd-Fe-B permanent magnetic alloy) were fixed above the GO membrane (Figure 1f) and the magnetic fields located at the GO membrane were measured by a magnetometer (TES, WT10A). During the penetration experiments, $80 \mathrm{~mL}$ of $0.1 \mathrm{~mol} / \mathrm{L}$ certain electrolytes and deionized water were injected into the source and drain vessels respectively and the conductivity variations of the drains were measured with time under mild mechanical stirring on a conductivity meter (INESA, DDS-307) to investigate the coexistence of concentration gradients and external fields on the ion transport behavior through GO membranes (Note: In the case of electric fields applied, the conductivity variations of the drains were measured after the voltages were tuned off to eliminate the effect of external electric fields on the conductivity measurement. After that, the voltages were tuned on again). In terms of the investigation on the control of ion fluidic flows through GO membranes under the applications of electric and magnetic fields, $80 \mathrm{~mL} 0.01 \mathrm{~mol} / \mathrm{L}$ certain electrolytes were injected into both the source and drain vessels with the same speed and the conductivity variations of both the sources and drains were measured with time under stirring. In the case of magneto-modulated transportations of $\mathrm{FeCl}_{3}$ sources through $\mathrm{GO}$ membranes, the $\mathrm{pH}$ values of the sources and drains were also measured with time on a pH meter (SANXIN, MP523) to study the penetration behavior of protons. After the penetration experiments, a simple hydrostatic pressure experiment was done to ensure the stability of the GO membranes in solutions. Briefly, one vessel of 

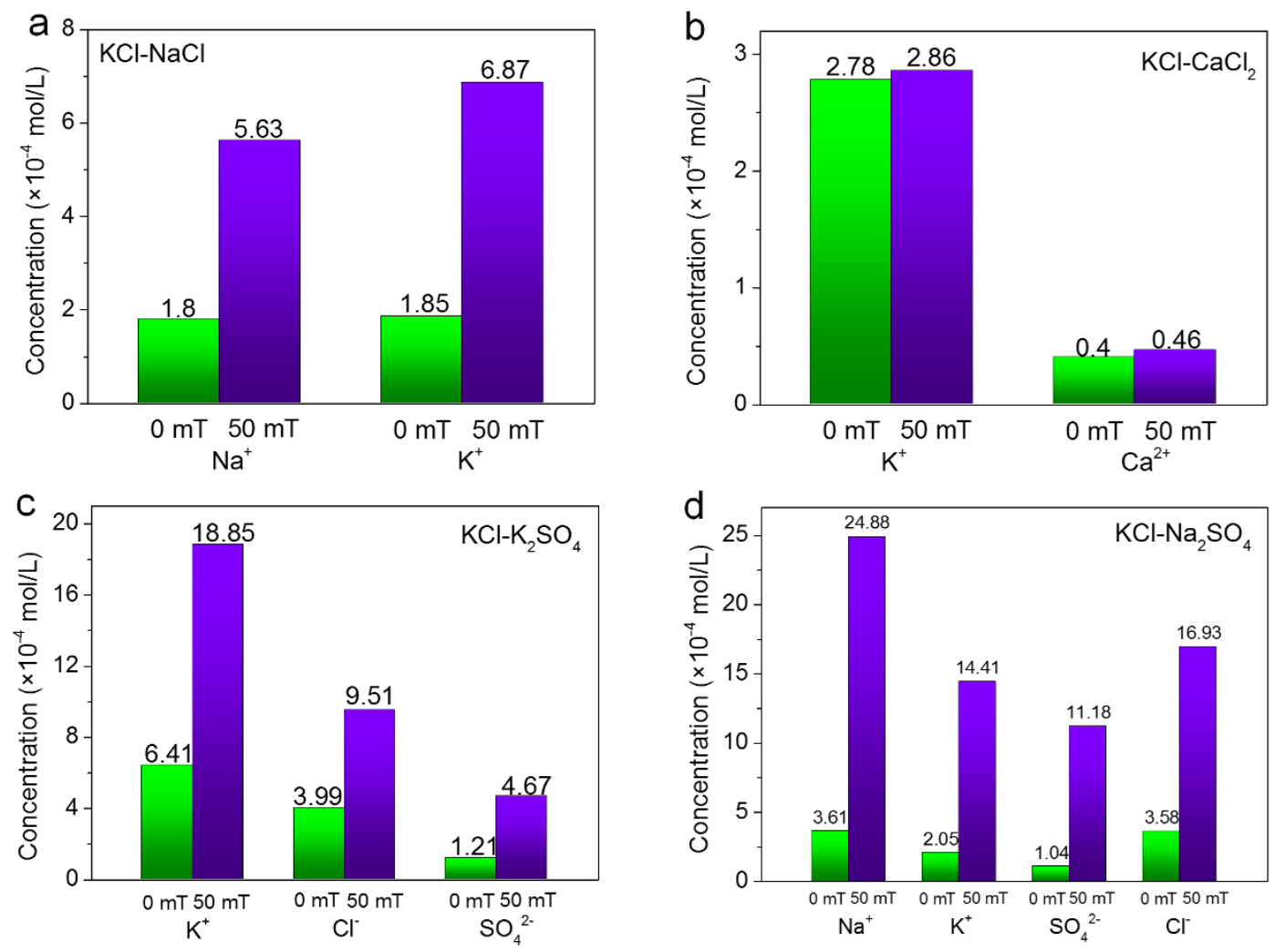

Figure 6 Ion concentrations of the drains during the penetrations of (a) $\mathrm{KCl}-\mathrm{NaCl}$, (b) $\mathrm{KCl}-\mathrm{CaCl}_{2},(\mathrm{c}) \mathrm{KCl}_{-} \mathrm{K}_{2} \mathrm{SO}_{4}$ and (d) $\mathrm{KCl}_{-} \mathrm{Na}_{2} \mathrm{SO}_{4}$ hybrid sources when applying magnetic fields (drain to source).

the penetration apparatus was filled with water while the other one was left empty. If no water flowed across the GO membrane during the 30 min of testing, it was believed that the GO membrane kept intact and stable during the penetration experiments.

Characterizations. The as-prepared GO sheets and GO membranes were characterized by atomic force microscopy (AFM, Agilent 5100), scanning electron microscope (SEM, LEO 1530,10kV) and Auger electron spectroscopy (AES, PHI 700). The magnetism of GO membranes was measured by SQUID magnetometer (Quantum Design MPMS-XL, USA). The concentrations of certain cations and anions in drains after $3 \mathrm{~h}$ of penetration were carried out by atomic emission spectroscopy (IRIS Intrepid II) and liquid chromatography (SY-5000), respectively.

1. Hou, X., Guo, W. \& Jiang, L. Biomimetic smart nanopores and nanochannels. Chem. Soc. Rev. 40, 2385-2401 (2011).

2. Bocquet, L. \& Charlaix, E. Nanofluidics, from bulk to interfaces. Chem. Soc. Rev. 39, 1073-1095 (2010).

3. Majumder, M., Chopra, N., Andrews, R. \& Hinds, B. J. Nanoscale hydrodynamics: Enhanced flow in carbon nanotubes. Nature 438, 44-44 (2005).

4. Holt, J. K. et al. Fast mass transport through sub-2-nanometer carbon nanotubes. Science 312, 1034-1037 (2006).

5. Fornasiero, F. et al. Ion exclusion by sub-2-nm carbon nanotube pores. Proc. Natl. Acad. Sci. U.S.A. 105, 17250-17255 (2008).

6. Choi, W. et al. Diameter-dependent ion transport through the interior of isolated single-walled carbon nanotubes. Nature Commun. 4, 2397 (2013).

7. Falk, K., Sedlmeier, F., Joly, L., Netz, R. R. \& Bocquet, L. Molecular origin of fast water transport in carbon nanotube membranes: Superlubricity versus curvature dependent friction. Nano Lett. 10, 4067-4073 (2010).

8. Joseph, S. \& Aluru, N. R. Why are carbon nanotubes fast transporters of water? Nano Lett. 8, 452-458 (2008).

9. Siria, A. et al. Giant osmotic energy conversion measured in a single transmembrane boron nitride nanotube. Nature 494, 455-458 (2013).

10. Logan, B. E. \& Elimelech, M. Membrane-based processes for sustainable power generation using water. Nature 488, 313-319 (2012).

11. Novoselov, K. S. et al. Electric field effect in atomically thin carbon films. Science 306, 666-669 (2004).

12. Kim, H. W. et al. Selective gas transport through few-layered graphene and graphene oxide membranes. Science 342, 91-95 (2013).

13. Li, H. et al. Ultrathin, molecular-sieving graphene oxide membranes for selective hydrogen separation. Science 342, 95-98 (2013).
14. Bunch, J. S. et al. Impermeable atomic membranes from graphene sheets. Nano Lett. 8, 2458-2462 (2008)

15. Koenig, S. P., Wang, L., Pellegrino, J. \& Bunch, J. S. Selective molecular sieving through porous graphene. Nature Nanotech. 7, 728-732 (2012).

16. Garaj, S., Liu, S., Golovchenko, J. A. \& Branton, D. Molecule-hugging graphene nanopores. Proc. Natl. Acad. Sci. U. S. A. 110, 12192 (2013).

17. Cohen-Tanugi, D. \& Grossman, J. C. Water desalination across nanoporous graphene. Nano Lett. 12, 3602-3608 (2012).

18. Stankovich, S. et al. Synthesis of graphene-based nanosheets via chemical reduction of exfoliated graphite oxide. Carbon 45, 1558-1565 (2007).

19. Eda, G., Fanchini, G. \& Chhowalla, M. Large-area ultrathin films of reduced graphene oxide as a transparent and flexible electronic material. Nature Nanotech. 3, 270-274 (2008).

20. Eda, G. \& Chhowalla, M. Chemically derived graphene oxide: Towards large-area thin-film electronics and optoelectronics. Adv. Mater. 22, 2392-2415 (2010).

21. Loh, K. P., Bao, Q., Eda, G. \& Chhowalla, M. Graphene oxide as a chemically tunable platform for optical applications. Nature Chem. 2, 1015-1024 (2010).

22. Nair, R. R., Wu, H. A., Jayaram, P. N., Grigorieva, I. V. \& Geim, A. K. Unimpeded permeation of water through helium-leak-tight graphene-based membranes. Science 335, 442-444 (2012).

23. Joshi, R. K. et al. Precise and ultrafast molecular sieving through graphene oxide membranes. Science 343, 752-754 (2014).

24. Sun, P. et al. Selective ion penetration of graphene oxide membranes. ACS Nano 7, 428-437 (2013).

25. Boukhvalow, D. W., Katsnelson, M. I. \& Son, Y. W. Origin of anomalous water permeation through graphene oxide membrane. Nano Lett. 13, 3930-3935 (2013).

26. Sun, P. et al. Selective trans-membrane transport of alkali and alkaline earth cations through graphene oxide membranes based on cation- $\pi$ interactions. ACS Nano 8, 850-859 (2014).

27. Picallo, C. B., Gravelle, S., Joly, L., Charlaix, E. \& Bocquet, L. Nanofluidic osmotic diodes: Theory and molecular dynamics simulations. Phys. Rev. Lett. 111, 244501 (2013).

28. Plecis, A., Schoch, R. B. \& Renaud, P. Ionic transport phenomena in nanofluidics: Experimental and theoretical study of the exclusion-enrichment effect on a chip. Nano Lett. 5, 1147-1155 (2005).

29. Vlassiouk, I. \& Siwy, Z. S. Nanofluidic diode. Nano Lett. 7, 552-556 (2007).

30. Daiguji, H., Oka, Y. \& Shirono, K. Nanofluidic diode and bipolar transistor. Nano Lett. 5, 2274-2280 (2005).

31. Karnik, R., Duan, C., Castelino, K., Daiguji, H. \& Majumdar, A. Rectification of ionic current in a nanofluidic diode. Nano Lett. 7, 547-551 (2007). 
32. Siwy, Z., Heins, E., Harrell, C. C., Kohli, P. \& Martin, C. R. Conical-nanotube ioncurrent rectifiers: The role of surface charge. J. Am. Chem. Soc. 126, 10850-10851 (2004).

33. Siwy, Z., Kosińska, I. D., Fuliński, A. \& Martin, C. R. Asymmetric diffusion through synthetic nanopores. Phys. Rev. Lett. 94, 048102 (2005).

34. He, J., Zhou, J., Lu, X. \& Corry, B. Bioinspired graphene nanopores with voltagetunable ion selectivity for $\mathrm{Na}^{+}$and $\mathrm{K}^{+}$. ACS Nano 7, 10148-10157 (2013).

35. Khurana, G., Kumar, N., Kotnala, R. K., Nautiyald, T. \& Katiyar, R. S. Temperature tuned defect induced magnetism in reduced graphene oxide. Nanoscale 5, 3346-3351 (2013).

36. Wang, Y. et al. Room-temperature ferromagnetism of graphene. Nano Lett. 9 , 220-224 (2009).

37. Ramakrishna Matte, H. S. S., Subrahmanyam, K. S. \& Rao, C. N. R. Novel magnetic properties of graphene: Presence of both ferromagnetic and antiferromagnetic features and other aspects. J. Phys. Chem. C 113, 9982-9985 (2009).

38. Yazyev, O. V. Magnetism in disordered graphene and irradiated graphite. Phys. Rev. Lett. 101, 037203 (2008).

39. Yazyev, O. V. \& Helm, L. Defect-induced magnetism in graphene. Phys, Rev. B 75, 125408 (2007).

40. Rao, C. N. R., Ramakrishna Matte, H. S. S., Subrahmanyam, K. S. \& Maitra, U. Unusual magnetic properties of graphene and related materials. Chem. Sci. 3, 45-52 (2012).

41. Rao, S. S., Jammalamadaka, S. N., Stesmans, A. \& Moshchalkov, V. V. Ferromagnetism in graphene nanoribbons: Split versus oxidative unzipped ribbons. Nano Lett. 12, 1210-1217 (2012).

42. Eng, A. Y. S. et al. Searching for magnetism in hydrogenated graphene: Using highly hydrogenated graphene prepared via birch reduction of graphite oxides. ACS Nano 7, 5930-5939 (2013).

43. Zhou, J. et al. Ferromagnetism in semihydrogenated graphene sheet. Nano Lett. 9, 3867-3870 (2009).

44. Kim, J. E. et al. Graphene oxide liquid crystals. Angew. Chem. Int. Ed. 50, 3043-3047 (2011).

45. Lee, S. H., Lee, D. H., Lee, W. J. \& Kim, S. O. Tailored assembly of carbon nanotubes and graphene. Adv. Funct. Mater. 21, 1338-1354 (2011).

46. Sun, P. et al. Magnetic transitions in graphene derivatives. Nano Res. DOI 10.1007/s12274-014-0512-1 (2014)
47. Joly, V. L. J. et al. Observation of magnetic edge state in graphene nanoribbons. Phys. Rev. B 81, 245428 (2010).

48. Karim, M. R. et al. Graphene oxide nanosheet with high proton conductivity. J. Am. Chem. Soc. 135, 8097-8100 (2013).

\section{Acknowledgments}

This work is supported by National Science Foundation of China (51372133), Beijing Science and Technology Program (D141100000514001), National Program on Key Basic Research Project (2014CB932401, 2011CB013000), and Beijing Natural Science Foundation (2122027)

\section{Author contributions}

H.W.Z. and P.Z.S.. conceived and designed the experiments. P.Z.S, F.Z. performed the experiments. M.L.Z., K.L.W. and D.H.W. conducted the theoretical analysis. P.Z.S. and H.W.Z. co-wrote the manuscript.

\section{Additional information}

Supplementary information accompanies this paper at http://www.nature.com/ scientificreports

Competing financial interests: The authors declare no competing financial interests.

How to cite this article: Sun, P. et al. Electro- and Magneto-Modulated Ion Transport through Graphene Oxide Membranes. Sci. Rep. 4, 6798; DOI:10.1038/srep06798 (2014).

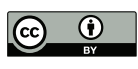

This work is licensed under a Creative Commons Attribution 4.0 International License. The images or other third party material in this article are included in the article's Creative Commons license, unless indicated otherwise in the credit line; if the material is not included under the Creative Commons license, users will need to obtain permission from the license holder in order to reproduce the material. To view a copy of this license, visit http://creativecommons.org/licenses/by/4.0/ 